\title{
A ROTATING CROSS-CYLINDER
}

BY

M. TREE

LONDON

THE astigmic lens or cross-cylinder was first described by Stokes in 1849 , but its popularisation and practical application to the methods of subjective testing of ametropia, is due to the insight of that "G.O.M." of American Ophthalmology, Dr. Edward Jackson.

The Jackson type of cross-cylinder is mounted in a fixed position with its handle at $45^{\circ}$ to the cylinder axes.

The manipulation of this cross-cylinder necessitates two procedures :-

1. Orientation of the cylinder axes, which implies rotation in a clockwise or anti-clockwise direction, in front of the trialframe which the patient is wearing.

2. Twirling of the cross-cylinder.

The act of twirling produces a rotation of the cross-cylinder axes through $90^{\circ}$. Dr. Jackson insisted that twirling gives absolute suddenness to the change of direction, and thus produces that maximum contrast which is necessary to help the patient in deciding on the combination which gives clearest visual acuity.

It is strange to note that in spite of the enthusiastic advocacy of the cross-cylinder by the celebrated Dr. Jackson, it is still not used with the frequency that it deserves. To some, no doubt, the action of the cross-cylinder is still wrapped in mystery, but I do not think that this is the entire explanation of its neglect. It has long seemed to me that although the mounting of the crosscylinder in a fixed position is useful to facilitate the act of twirling, it introduces grave inconvenience where orientation of the cylinder axes is concerned. The act of rotating the Jackson cross-cylinder is apt on occasion to become an awkward procedure, which is irritating both to patient and oculist alike. If the handle is not to abut on the patient's nose, it is equally liable to become entangled in the knobs and adjusting mechanisms of the modern trial-frame.

To overcome these inconveniences, Messrs. Theodore Hamblin have constructed a rotating cross-cylinder, to my specification, which is here illustrated. The handle is mounted at right angles to a semi-circular scale which is marked in degrees from $0^{\circ}$ to $180^{\circ}$ as on the ordinary trial frame. The cross-cylinder lens is mounted within the semi-circular scale, and is rotated in it by means of a cog-wheel situated at the upper part of the handle. 


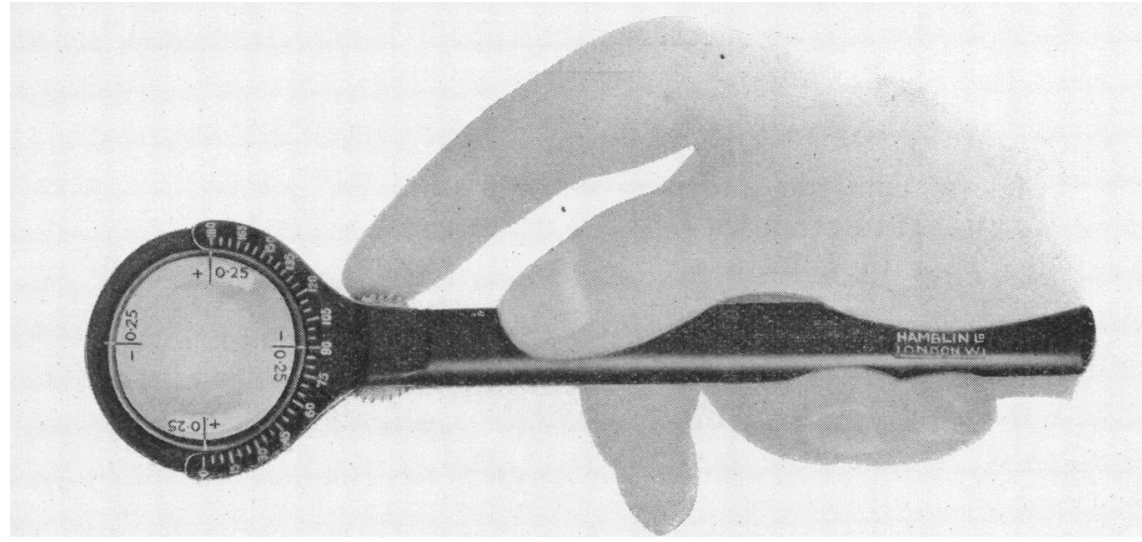

During orientation of the cross-cylinder, the handle is held vertically in front of the trial-frame worn by the patient. The lens is rotated into its optimum position by means of the cogwheel, which is turned by the examiner's index finger, and the handle is meanwhile retained steadily in the vertical position. The exact positions, in degrees, of the cross-cylinder axes are at once apparent on the scale. The next step is to rotate the whole cross cylinder lens through $90^{\circ}$-which is the procedure comparable to twirling the original Jackson cross-cylinder. This rotation through $90^{\circ}$ may be accomplished in several ways :-

1. The cog-wheel may be used and the lens quickly and suddenly rotated. This is facilitated by the fact that the new position of the appropriate axis is already indicated on the scale, by the position of the meridian of the opposite cylindrical component.

2. Instead of using the cogwheel, the handle may be swung outwards from the vertical to the horizontal position.

3 . In the special circumstances, where on orientation, the cylinder axes are at $45^{\circ}$ to the handle, i.e., with the axes at $45^{\circ}$ and $135^{\circ}$ the handle may be twirled as with the Jackson cross-cylinder.

I am quite convinced that by either of the first two methods the cylindrical lens can be rotated through $90^{\circ}$ with sufficient suddenness to provide the abrupt contrast necessary. It is true that the act of twirling an orientated Jackson cross-cylinder may give serene pleasure to the joyful oculist and perhaps impart the impression of some magical sleight-of-hand; yet it should not be forgotten that the net effect is simply to rotate a lens through $90^{\circ}$. I venture to suggest that this pleasure is so often outweighed by the preceding difficulties, that the adoption of a rotating type of cross-cylinder, such as I have above described, will by its simplicity and ease of manipulation conduce to the more extended use of the cross-cylinder. 